\title{
RATIO-BASED SIMILARITY CRITERIA FOR POLARIMETRIC SAR IMAGE
}

\author{
H.Aghababaei ${ }^{1}$, G. Ferraioli ${ }^{2}$, V. Pascazio ${ }^{3}$ \\ ${ }^{1}$ Department of Earth Observation Science, University of Twente, Faculty of Geo-Information Science and Earth Observation, \\ Twente, The Netherlands \\ ${ }^{2}$ Dipartimento di Scienze e Tecnologie, Università degli Studi di Napoli "Parthenope", Napoli, Italy. \\ ${ }^{3}$ Dipartimento di Ingegneria, Università degli Studi di Napoli "Parthenope," Napoli, Italy.
}

KEY WORDS: Polarimetric SAR image, Similarity criterion, Non Local Filters, Ratio Image

\begin{abstract}
:
Dealing with multi-look polarimetric synthetic aperture radar (PolSAR) images requires averaging several independent looks to generate a sample covariance matrix of similar target scattering vectors. Along this, estimation of optimal similarity between target scattering vectors is still an open issue. In the literature, this intrinsic task has been mainly addressed in the information-based, geometric-based and detection-based frameworks. However, the derived measures mainly rely on the model assumption such as fully developed speckle and circular complex Gaussian distribution of the scattering vectors, which may not be held in high-resolution images of urban environments. To cope with this possible issue a discriminative model-free measure is proposed, where the similarity of target scattering is computed in the framework of non-local or patch based algorithm. In particular, the discriminative measure is constructed using the ratio between two pre-estimated covariance matrices of the scattering vectors. Experimental validation of the proposed measure is provided using ALOS-PALSAR image and compared with existing criterions in the literature.
\end{abstract}

\section{INTRODUCTION}

Polarimetric synthetic aperture radar (PolSAR) systems make use of different polarizations at emission and reception of the radar wave provides a deeper insight into the backscattering mechanisms. The target scattering vector $\mathbf{k}$ can be formed by stacking of the complex values corresponding to each particular polarization channel representing the backscattering mechanism of the target under analysis.

Polarimetric SAR data provides useful information on different terrain applications from classification to change detection. The core of the most PolSAR information extraction algorithms is related to the proper identification of similar pixels or patches. However, the coherent synthetic aperture radar (SAR) images are affected by the well-known multiplicative speckle noise [1]. The speckle may increase the intensity fluctuations and severely diminish the performance of information extraction techniques in SAR image as well as in the applications requiring multi-channel polarimetric SAR data sets. Typically in PolSAR image, the fluctuations related to the speckle are usually signal independent while they are correlated between polarization channels and might be dependent on the scattering properties of the observed targets [2]. Accordingly, finding the similar pixels in PolSAR images with noisy target scattering vectors is a complicated task. In the frame of PolSAR data, the conventional speckle reduction techniques are based on an adaptive selection of the pixels that follow similar polarimetric target scattering mechanisms [2, 3]. However, recently the attention of SAR research community has been drawn into the use of nonlocal (NL) or patch means filters. The basic principle of NL filter is moving from local neighboring pixels to non-local similar pixel and restoring pixel value by exploiting similar pixels in the image, where those usually detected by a searching window around the selected pixel. It is known that the weighted averaging of similar pixels can effectively suppress the speckle with perceptible preservation of structures and the textures. However, the most critical aspect of NL approaches returns to the decision of choosing similar pixel. This is mainly performed through a similarity or dissimilarity criterion, where it quantifies how much the two specific patches are similar or different. It is clear that the similarity between two patches can be defined as the sum of the similarity of each pair of corresponding pixels in the two noisy patches in the image. Then, if two noisy patches are similar according to the defined criteria, then the central pixels of the patches are used together in the filtering process.

In literature, several criterions have been proposed and evaluated for computing the (dis)similarity of noisy patches in polarimetric SAR images. The different criterions, including informationbased, geometric-based and detection-based frameworks, have been proposed in relation to the different application of PolSAR image including filtering and change detection. For more information about the different criterions, the reader may refer to the works in $[4,5]$.

However, the developed criterions in the literature mainly rely on a fundamental Goodman's fully developed speckle model and circular complex Gaussian distribution of the scattering vectors, where it may not be held in high-resolution images of urban environments, in which persistent scatterers and not fully developed speckle are frequently encountered. In such a case, when the estimated criterion accounts for the particular speckle model, the efficiency of NL based filtering can be severely impaired. Hence, the model-free approaches of comparing noisy patches in polarimetric SAR image that is sorely lacking in the literature would be very useful.

In this paper, to cope with the above-mentioned issue and enhance the capability of non-local approaches, a model-free technique is proposed, where the similarity in PolSAR image is defined based on the ratio between the patch containing the reference pixel and the patch containing the target pixel. In particular, the Kolmogorov-Smirnov distance (KSD) between cumulative distribution functions of the ratio-patch and inverse ratio-patch defines the employed model-free similarity measure.

The next section of the paper presents the framework of the proposed non-local ratio based dissimilarity criterion. Then, the paper is followed by some experimental results and validation through the comparison with most existing competitive similarity measures in Section 3. 


\section{THE RATIO BASED DISSIMILARITY MEASURE IN POLSAR DATA}

Fully polarimetric SAR sensors in the linear horizontal and vertical polarization basis measures the $2 \times 2$ scattering matrix as:

$$
\mathbf{S}=\left[\begin{array}{ll}
S_{H H} & S_{H V} \\
S_{V H} & S_{V V}
\end{array}\right]
$$

where $H$ and $V$ represent the horizontal and vertical linear polarizations, respectively. Moreover, in case of the reciprocal backscattering, i.e. $\mathrm{S}_{\mathrm{HV}}=\mathrm{S}_{\mathrm{VH}}$, the information of scattering matrix can be represented using a $3 \times 1$ complex vector, known as target scattering vector, as: $\mathbf{k}=\left[\begin{array}{lll}\mathrm{S}_{\mathrm{HH}} & \sqrt{2} \mathrm{~S}_{\mathrm{HV}} & \mathrm{S}_{\mathrm{VV}}\end{array}\right]^{\mathrm{T}}$, where ${ }^{\mathrm{T}}$ indicates the transpose operator.

It is known that under the assumption of fully developed speckle and homogeneity of elementary scatterers inside a resolution cell, the so-called target scattering vector $\mathbf{k}$ follows a 3-dimensional circular complex Gaussian distribution as [6]:

$$
p(\mathbf{k} \mid \Sigma)=\frac{1}{\pi^{3}|\Sigma|} \exp \left(-\mathbf{k}^{\dagger} \Sigma^{-1} \mathbf{k}\right)
$$

where $\sum=\mathrm{E}\left\{\mathbf{k k}^{\dagger}\right\}$ is $3 \times 3$ complex covariance matrix. The operator $\mathrm{E},|\mathbf{X}|$, and ${ }^{\dagger}$ denote the mathematical expectation, the determinant of matrix $\mathbf{X}$, and the Hermitian transpose, respectively.

Multi-look polarimetric SAR processing usually requires averaging several independent looks to generate a sample covariance matrix of $L$ similar target scattering vectors.

$$
\mathbf{C}=\frac{1}{L} \sum_{l=1}^{L} \mathbf{k}_{l} \mathbf{k}_{l}^{\dagger}
$$

In (3), apparently the sum is carried over several scattering vectors, $\mathbf{k}_{l}, l=1,2, \ldots, L$, for each pixel. Alongside with the above assumption and when $L \geq 3$, the empirical covariance $\mathbf{C}$ follows a Wishart distribution given by:

$$
p(\mathbf{C} \mid \Sigma, L)=\frac{L^{3 L}|\mathbf{C}|^{L-3}}{\Gamma_{3}(L)|\Sigma|^{L}} \exp \left(-L \operatorname{tr}\left(\Sigma^{-1} \mathbf{C}\right)\right)
$$

where $\operatorname{tr}($.$) is the matrix trace.$

In the framework of non-local approaches, the estimation of the dissimilarity between two specific pixels, namely pixel $p$ and pixel $q$, starts from the pre-estimation of sample covariance matrices $\hat{\mathbf{C}}$ in (3). The pre-estimation of covariance matrices is later employed within the patches around the considered pixels in order to compute their dissimilarity. In this case, the output of the classical local based or adaptive methods can be used as a pre-estimation of covariance matrix. The pre-estimation can also mitigate the large fluctuations in the polarimetric image. Having the pre-estimated covariance matrix for each pixel in the image, then two patches around reference pixel $p$ and target pixel $q$, i.e. $\mathbf{X p}$ and $\mathbf{X q}$ with same size is considered, i.e. $\mathbf{X p}=\left\{\mathbf{C}\left(\mathbf{p}_{1}\right)\right.$, $\left.\mathbf{C}\left(\mathrm{p}_{2}\right), \ldots, \mathbf{C}\left(\mathrm{p}_{\mathrm{N}}\right)\right\}$ and $\mathbf{X q}=\left\{\mathbf{C}\left(\mathrm{q}_{1}\right), \mathbf{C}\left(\mathrm{q}_{2}\right), \ldots, \mathbf{C}\left(\mathrm{q}_{\mathrm{N}}\right)\right\}$, where $N$ is total number of pixels in the patches.

In this case, the similarity between two patches can be defined as the sum of the similarity of each pair of corresponding pixels obtained by the comparison of their corresponding pre-estimated covariance matrices.

In literature, the (dis)similarity was defined in different frameworks [4]. Following the assumption of Wishart distribution of $\mathbf{C}$, in the frame of detection approach, dissimilarity can be defined based on the detection of the difference between the underlying $\mathbf{C}\left(\mathrm{p}_{\mathrm{n}}\right)$ and $\mathbf{C}\left(\mathrm{q}_{\mathrm{n}}\right)$. Along this, the generalized likelihood ratio (GLR) leads to a criterion defined as the following log ratio [7]:

$$
\begin{aligned}
& \delta_{n}\left(\mathbf{C}\left(p_{n}\right), \mathbf{C}\left(q_{n}\right)\right)=2 L \log \frac{\frac{1}{2}\left|\mathbf{C}\left(p_{n}\right), \mathbf{C}\left(q_{n}\right)\right|}{\sqrt{\left|\mathbf{C}\left(p_{n}\right)\right| \mathbf{C}\left(q_{n}\right) \mid}} \\
& \Delta^{G L R}=\frac{1}{N} \sum_{n} \delta_{n}\left(\mathbf{C}\left(p_{n}\right), \mathbf{C}\left(q_{n}\right)\right)
\end{aligned}
$$

Similarly, the symmetrical version of Kullback-Leibler divergence $\Delta^{\mathrm{SKL}}$ and the geodesic and Riemannian distance $\Delta^{\mathrm{Geo}}$ in the case of Wishart-distributed empirical covariance matrices can be represented as:

$$
\begin{aligned}
& \delta_{n}\left(\mathbf{C}\left(p_{n}\right), \mathbf{C}\left(q_{n}\right)\right)=\operatorname{Ltr}\left(\mathbf{C}^{-1}\left(p_{n}\right) \mathbf{C}\left(q_{n}\right)+\mathbf{C}^{-1}\left(q_{n}\right) \mathbf{C}\left(p_{n}\right)\right) \\
& \Delta^{K S L}=\frac{1}{N} \sum_{n} \delta_{n}\left(\mathbf{C}\left(p_{n}\right), \mathbf{C}\left(q_{n}\right)\right)
\end{aligned}
$$

and

$$
\begin{aligned}
& \delta_{n}\left(\mathbf{C}\left(p_{n}\right), \mathbf{C}\left(q_{n}\right)\right)=\left\|\log \left(\mathbf{C}^{-1 / 2}\left(q_{n}\right) \mathbf{C}\left(p_{n}\right) \mathbf{C}^{-1 / 2}\left(q_{n}\right)\right)\right\| \\
& \Delta^{G e o}=\frac{1}{N} \sum_{n} \delta_{n}\left(\mathbf{C}\left(p_{n}\right), \mathbf{C}\left(q_{n}\right)\right)
\end{aligned}
$$

where $\log$ is the matrix logarithm and $\|$.$\| is the Froebenius norm.$

It is apparent the above dissimilarity measures rely on the aforementioned models in (2) to (4). Accordingly, their efficiency may be impaired in urban environment and in the situation where the assumptions may not be valid.

In this paper in order to properly discriminate the dissimilar pixels, the similarity between two noisy patches is computed through the KSD between empirical cumulative distributions of the trace $(T r)$, the Maximum $\left(\lambda_{\max }\right)$ and the Minimum $\left(\lambda_{\min }\right)$ Eigenvalue of the ratio $\mathbf{R}$ and inverse ratio $\mathbf{I R}$ matrices generated as follows.

$$
\begin{aligned}
& \mathbf{R}\left(p_{n}, q_{n}\right)=\mathbf{C}^{-1 / 2}\left(q_{n}\right) \hat{\mathbf{C}}\left(p_{n}\right) \hat{\mathbf{C}}^{-1 / 2}\left(q_{n}\right) \\
& \operatorname{IR}\left(p_{n}, q_{n}\right)=\mathbf{C}^{-1 / 2}\left(p_{n}\right) \hat{\mathbf{C}}\left(q_{n}\right) \hat{\mathbf{C}}^{-1 / 2}\left(p_{n}\right)
\end{aligned}
$$

Accordingly, the proposed measures can be given as:

$$
\begin{aligned}
& \Delta^{T r}=\frac{1}{N} \sum_{n} K S D\left(\operatorname{Tr}\left(\mathbf{R}\left(p_{n}, q_{n}\right)\right), \operatorname{Tr}\left(\mathbf{I R}\left(p_{n}, q_{n}\right)\right)\right) \\
& \Delta^{\lambda_{\min }}=\frac{1}{N} \sum_{n} K S D\left(\lambda_{\min }\left(\mathbf{R}\left(p_{n}, q_{n}\right)\right), \lambda_{\min }\left(\mathbf{I R}\left(p_{n}, q_{n}\right)\right)\right) \\
& \Delta^{\lambda_{\max }}=\frac{1}{N} \sum_{n} K S D\left(\lambda_{\max }\left(\mathbf{R}\left(p_{n}, q_{n}\right)\right), \lambda_{\max }\left(\mathbf{I R}\left(p_{n}, q_{n}\right)\right)\right)
\end{aligned}
$$

\section{EXPERIMENTAL RESULTS}

In this section, the performance of the proposed dissimilarity measures in (9) is evaluated and analysed using a real polarimetric data set acquired by ALOS-PALSAR sensor over San Francisco, Bye area in 21th Aguste 2018. The Pauli colour 
coded of the selected region with size of $400 \times 500$ pixels is shown in Figure 1. The evaluation process is performed in three different scenarios. In particular, three reference pixels correspond to urban area $(\operatorname{pixel} A)$, vegetation area $($ pixel $B)$ and rough surface area (pixel $C$ ) are considered and the similarity of all other pixels in the image to each of these reference pixels is computed. The similarity is computed using the proposed measures as well as the existing features in the literature given in the Eq.(5) (7). In addition, to the aim of fair comparison, the estimated similarity images are normalized in the range of $[0,1]$, where pixels with value of zero indicating the high similarity with considered reference pixel, while the value 1 is related to pixels that are mainly different from the reference pixel. The results by each scenarios are given in Figures 2-4. All the similarity measures were computed using a patch with size of $7 \times 7$.

As can be seen in all scenarios, where the reference target is chosen in urban (Fig. 2), vegetation (Fig. 3) or in surface area (Fig. 4), the estimated similarity by the proposed method significantly outperforms the other existing approaches. The lower performance of the similarity measures given in Eq.(5) (7), mainly is due to the considered assumptions in the target scattering vector and covariance matrix models that may not be valid in the textured non-homogeneous areas. Instead, the proposed approach is a model-free dissimilarity measure and can be valid in general cases. The reason for the efficiency of the proposed method returns to the fact that the speckle has multiplicative characteristic in SAR images. In a scenario when the deterministic components of the two considered patches are equal for the same position in the patches, then the ratio and inverse ratio between two noisy patches is equal to the ratio between the speckled patches. In such a case, the trace or Eigenvalue distributions from ratio $\mathbf{R}$ and inverse ratio IR should be same or as much as possible close to each other.

Comparing the three proposed features $\left(\lambda_{\max }, \lambda_{\min }, T r\right)$ shows that those are very close to each other, specifically when the reference target is chosen in the vegetation and rough surface areas. However, when the reference pixel is selected in the urban area some differences between $\lambda_{\max }$ and $\left(\lambda_{\min }\right.$ and $\left.T r\right)$ were observed. This might be related to the high backscattering power of urban area with respect to other regions in the image, which showed its effect in the estimated $\lambda_{\max }$ dissimilarity measure.

The proposed dissimilarities can be evaluated in the framework of polarimetric SAR image filtering, where the proposed similarity is employed to find similar target scattering vectors in order to properly estimate the polarimetric sample covariance matrix.

\section{REFERENCES}

J. W. Goodman, "Some fundamental properties of speckle*," Journal of the Optical Society of America, vol. 66, no. 11, pp. 1145-1150, 1976/11/01 1976.

J.-S. Lee and E. Pottier, Polarimetric radar imaging: from basics to applications. CRC press, 2009.

L. Jong-Sen, M. R. Grunes, and G. d. Grandi, "Polarimetric SAR speckle filtering and its implication for classification," IEEE Transactions on Geoscience and Remote Sensing, vol. 37, no. 5, pp. 2363-2373, 1999.

C. Deledalle, L. Denis, G. Poggi, F. Tupin, and L. Verdoliva, "Exploiting Patch Similarity for SAR Image Processing: The nonlocal paradigm," IEEE

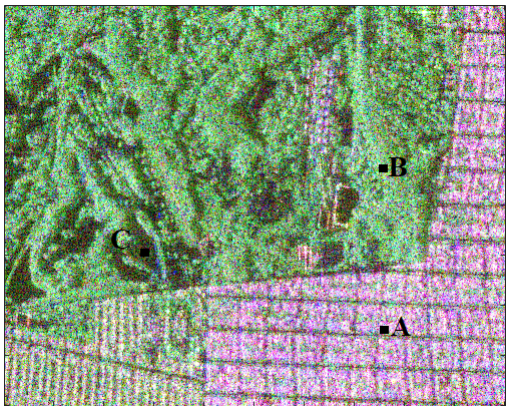

Figure 1. Pauli colour coded image of study area

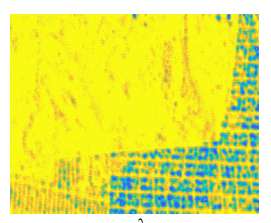

(a) $\Delta^{\lambda \max }$

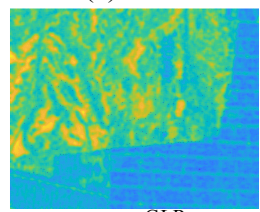

(d) $\Delta^{\mathrm{GLR}}$

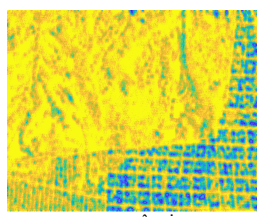

(b) $\Delta^{\lambda \min }$

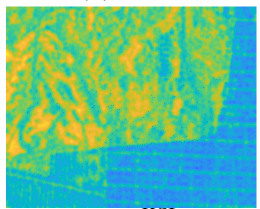

(e) $\Delta^{\mathrm{KSL}}$

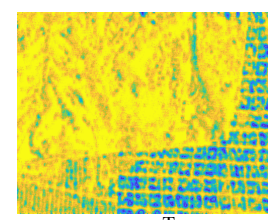

(c) $\Delta^{\operatorname{Tr}}$

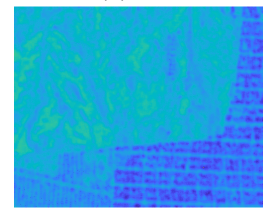

(f) $\Delta^{\text {Geo }}$
Figure 2. similarity of the image to the specified target A

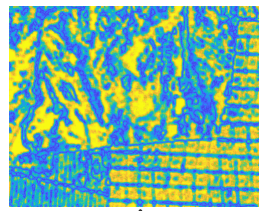

(a) $\Delta^{\lambda \max }$

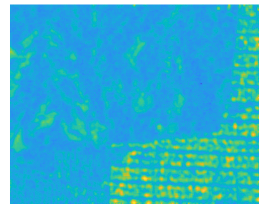

(d) $\Delta^{\mathrm{GLR}}$

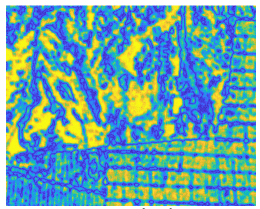

(b) $\Delta^{\lambda \min }$

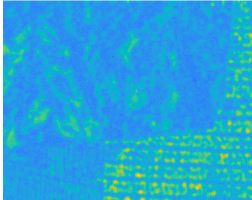

(e) $\Delta^{\mathrm{KSL}}$

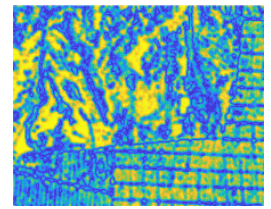

(c) $\Delta^{\operatorname{Tr}}$

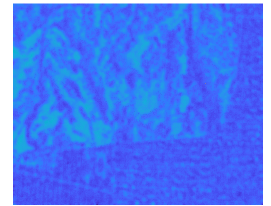

(f) $\Delta^{\text {Geo }}$ \begin{tabular}{cccccccccc}
\hline & 0.1 & 0.2 & 0.3 & 0.4 & 0.5 & 0.6 & 0.7 & 0.8 & 0.9
\end{tabular}

Figure 3. similarity of the image to the specified target B

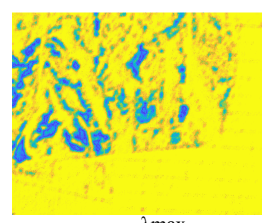

(a) $\Delta^{\lambda \max }$

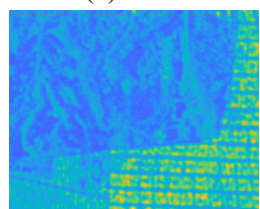

(d) $\Delta^{\mathrm{GLR}}$

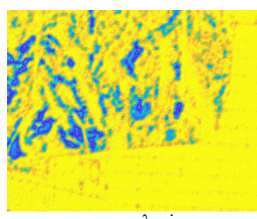

(b) $\Delta^{\lambda \min }$

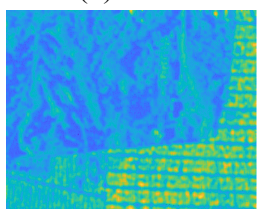

(e) $\Delta^{\mathrm{KSL}}$

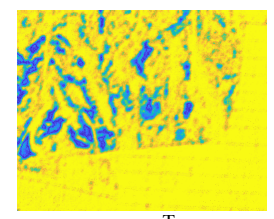

(c) $\Delta^{\operatorname{Tr}}$

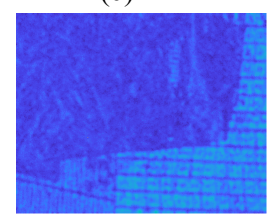

(f) $\Delta^{\text {Geo }}$

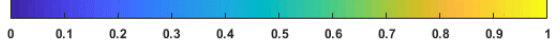

Figure 4. similarity of the image to the specified target C 
Signal Processing Magazine, vol. 31, no. 4, pp. 69-78, 2014.

[5] C.-A. Deledalle, L. Denis, and F. Tupin, "How to compare noisy patches? Patch similarity beyond Gaussian noise," International journal of computer vision, vol. 99, no. 1, pp. 86-102, 2012.

[6] N. R. Goodman, "Statistical analysis based on a certain multivariate complex Gaussian distribution (an introduction)," The Annals of mathematical statistics, vol. 34, no. 1, pp. 152-177, 1963.

[7] K. Conradsen, A. A. Nielsen, J. Schou, and H. Skriver, "A test statistic in the complex Wishart distribution and its application to change detection in polarimetric SAR data," IEEE Transactions on Geoscience and Remote Sensing, vol. 41, no. 1, pp. 4-19, 2003. 